\title{
SOME FRIEDMANN COSMOLOGICAL SOLUTIONS IN THE SCALE COVARIANT THEORY OF GRAVITATION
}

\author{
AROONKUMAR BEESHAM \\ Department of Applied Mathematics \\ University of Zululand \\ Private Bag X1001, Kwa-Dlangezwa 3886, South Africa \\ (Received December 2, 1986)
}

ABSTRACT. The scale covariant theory of gravity admits the possibility of a time varying gravitational constant but contains a gauge function for which there is no independent equation. The circumstances under which explicit forms for a gauge function may be derived within the context of Friedmann-Robertson-Walker cosmological models are investigated and several forms are derived.

KEY WORDS AND PHRASES. Alternative gravitational theories, scale covariant theory, cosmology, Friedmann models.

1980 AMS SUBJECT CLASSIFICATION CODES. 83D, 83F.

\section{INTRODUCTION.}

It is well known that general relativity does not admit a time varying gravitational constant $G$. Numerous attempts to study such a variation of $G$ within general relativity have not proved successful [1-3]. The scale covariant theory of Canuto et al [4] is a viable scalar tensor theory which allows for a varying $G[5,6]$. The field equations in this theory for zero cosmological constant are [7].

$$
\mathrm{R}_{\mathrm{ab}}-\frac{1}{2} \mathrm{Rg}_{\mathrm{ab}}+\mathrm{f}_{\mathrm{ab}}(\beta)=\mathrm{GT}_{\mathrm{ab}}
$$

where the function $f_{a b}$ is given by

$$
\beta^{2} \mathrm{f}_{\mathrm{ab}}=2 \beta \beta_{\mathrm{a} ; \mathrm{b}}-4 \beta_{\mathrm{a}} \beta_{\mathrm{b}}-\left(2 \beta \beta_{i c}^{c}-\beta_{\mathrm{c}} \beta^{c}\right) \mathrm{g}_{\mathrm{ab}} .
$$

In equation (1), $R_{a b}$ is the Ricci tensor, $R$ the Ricci scalar and $T_{a b}$ the energy momentum tensor. We are using units in which the speed of light is unity and the constant $8 \pi$ has been absorbed into G. The gauge function $\beta$ is a scalar function satisfying $0<\beta<\infty$. Latin indices takes values 0 to 3. A semi-colon denotes covariant derivative and we are using the notation $\beta_{\mathbf{a}}$ for the ordinary derivative of $\beta$ with respect to $\mathrm{x}^{\mathrm{a}}$. For further details of the signs and conventions we are using see Ellis [8]. 
A. pecular feature of the scale covariant theory is that no independent equation for $\beta$ exists (see also [9]). The normal procedure is to adopt an equation of the form [11]

$$
\beta(t)=\left(t_{0} / t\right) \epsilon= \pm \frac{1}{2}, \pm 1, t_{0}=\text { constant }
$$

and then to try to determine which form best fits the observations and experimental results. However there are certain instances in which we may determine explicit forms for $\beta$. We shall investigate when this is possible within the context of Friedmann-Robertson-Walker cosmological models and then determine some explicit forms for $\beta$.

\section{FIELD EQUATIONS.}

The universe appears spatially homogeneous and isotropic about us. This leads to the Robertson-Walker metric which in comoving coordinates has the form

$$
d s^{2}=-d t^{2}+R^{2}(t)\left[d r^{2} /\left(1-k r^{2}\right)+r^{2} d \theta^{2}+r^{2} \sin ^{2} \theta d \phi^{2}\right],
$$

where $k= \pm 1$ or 0 . We assume the perfect fluid form for the energy momentum tensor

$$
\mathrm{T}_{\mathrm{ab}}=\left(\mu+\mathrm{p}_{\mathrm{a}} \mathrm{u}_{\mathrm{a}} \mathrm{u}_{\mathrm{ab}}+\mathrm{pg}_{\mathrm{a}}\right.
$$

where $\mu$ and $\mathrm{p}$ are, respectively, the energy density and pressure of the fluid, $u_{a}$ is the fluid four-velocity and $\mathrm{g}_{\mathrm{ab}}$ is the metric tensor. In the comoving coordinates we are using, $\mathrm{u}_{\mathrm{a}}$ has the form $u^{a}=\delta_{0}^{a}$. The field equations (1) and (2) become, for the Robertson-Walker metric (4) and the energy momentum tensor (5) [4]

$$
\begin{gathered}
3 \ddot{\mathrm{R}} / \mathrm{R}+3 \ddot{\beta} / \beta+3 \ddot{\mathrm{R}} \dot{\beta} /(\mathrm{R} \beta)=\frac{1}{2} \mathrm{G}(\mu+3 \mathrm{p}), \\
3(\dot{\mathrm{R}} / \mathrm{R}+\dot{\beta} / \beta)^{2}+3 \mathrm{k} / \mathrm{R}^{2}=\mathrm{G} \mu,
\end{gathered}
$$

where the overhead dot denotes a derivative with respect to time.

Assuming an equation of state of the form

$$
\mathrm{p}=(\gamma-1) \mu, 1 \leq \gamma \leq 2,
$$

equations (6) and $(7)$ lead us to the following conservation equation

This equation may readily be integrated to yield

$$
\mu+3 \gamma \mu \dot{\mathrm{R}} / \mathrm{R}=-\mu(\mathrm{G} \beta) \cdot /(\mathrm{G} \beta)-3(\gamma-1) \dot{\beta} / \beta .
$$

$$
\mathrm{G} \mu=1 /\left(\mathrm{R}^{3} \gamma \beta^{3 \gamma-2}\right) \text {. }
$$

We have here two independent equations (7) and (9) but four unknowns $R, \beta, G$ and $\mu$. Canuto and his coworkers attempted to solve these equations by assuming equation (3) for $\beta$ and also the condition $G^{-1 / t}$, which is a consequence of Dirac's large numbers hypothesis [11]. Since observations have indicated that a variation of $\mathrm{G}^{-} 1 / \mathrm{t}$ is not likely (Hellings et al [12]), we do not assume this condition. Rather we shall seek static solutions and show that they lead to explicit forms for $\beta$. Static solutions are important in the scale covariant theory because nonstatic Friedmann solutions in general relativity could appear to be static in the scale covariant theory [13].

Substituting equation (9) into equation (7), we obtain

$$
3(\dot{\mathrm{R}} / \mathrm{R}+\dot{\beta} / \beta)^{2}+3 \mathrm{k} / \mathrm{R}^{2}=1 /\left(\mathrm{R}^{3 \gamma} \beta^{3 \gamma-2}\right) \text {. }
$$

For static solutions $R=0, R=R_{0}=$ constant, and equation (10) becomes

$$
3 \beta^{2} / \beta^{2}+3 \mathrm{k} / \mathrm{R}_{0}^{2}=1 /\left(\mathrm{R}_{0}^{3 \gamma} \beta^{3 \gamma-2}\right) \text {. }
$$

We now discuss the solutions to equation (11). 
3. SOLUTIONS FOR $k=0$

The solutions can be written in the compact form

$$
\beta=\left[\frac{(3 \gamma-2) t}{2 \sqrt{3 R_{0}^{3 \gamma}}}\right]^{2 /(3 \gamma-2)}
$$

4. SOLUTIONS FOR $\mathrm{k}=+1$

(i) Dust $(\gamma=1)$

$$
\beta=\left[1-\cos \left(t / R_{0}\right)\right] /\left(6 R_{o}\right) .
$$

(ii) Radiation $(\gamma=4 / 3)$

(iii) Stiff matter $(\gamma=2)$

$$
\beta=\left[\sin \left(t / R_{0}\right)\right] / R_{0}
$$

$$
\beta=\left[\sin 2 t /\left(\sqrt{3} R_{0}{ }^{4}\right)\right]^{\frac{1}{2}} .
$$

5. SOLUTIONS FOR $\mathrm{k}=-1$

(i) Dust $(\gamma=1)$

$$
\beta=\left[\cosh \left(t / R_{o}\right)\right] /\left(6 R_{o}\right)-1 /\left(6 R_{o}\right) .
$$

(ii) Radiation $(\gamma=4 / 3)$

$$
\beta=\left[\sinh \left(t / R_{0}\right)\right] /\left(\sqrt{3} \mathrm{R}_{0}\right) .
$$

(iii) Stiff matter $(\gamma=2)$

$$
\beta=\left[\sinh \left(2 t / R_{0}\right)\right] /\left(3 R_{0}^{2}\right) .
$$

\section{DISCUSSION}

In each of the above solutions we have chosen a constant of integration to be zero so that $\beta(0)=0$. The solution (12) for $\gamma=1$ has also been given by Rahman and Banerii [13]. Our solutions can also be obtained by transformations from the nonstatic Friedmann-RobertsonWalker solutions in general relativity given by Vajk [14]. (Note that the $k=+1$ case of Vajk corresponds to our $k=-1$ case and vice versa). It is these nonstatic solutions which would appear to be static in the scale covariant theory.

It is clear from our solutions and equation (9) that $G \mu$ varies with time even though the solutions are static (see also [13]). This is not possible in the case of static cosmological models in general relativity.

The scale covariant theory is a generalization of general relativity. The procedure for obtaining corresponding general relativistic solutions (when they exist) from those in the scale covariant theory is to put $\beta=1$ and $G=$ constant $[4,13]$. Our solution (13) reduces under this procedure to the static Einstein universe in general relativity (see [8] for the latter). Apart from this specific solution, our other solutions have no corresponding static general relativistic analogues.

Explanation of the observed red shifts of galaxies cannot be given in the conventional way as due to recession of galaxies. However the main importance of the presented solutions is not so much their viability but in the forms of the gauge function.

Finally, we remark that the nonstatic-Friedmann-Robertson-Walker models in the scale covariant theory have been studied by Cariuto et al [10] (the dust and radiation cases) and by us [15] (the stiff matter case). In these solutions the gauge function appears as an arbitrary function. 
By putting $\beta=1$ and $\mathrm{G}=$ constant, the general relativistic solutions given by Vajk which we mentioned earlier are obtained.

As far as the physical interpretation of our solutions is concerned, we note that whilst in the gravitational units of general relativity the universe appears to be expanding, in the atomic units of the scale covariant theory, the universe could appear to be static [13]. Furthermore, for theoretical reasons, the study of static universes is appealing [16]. The value of the Hubble constant has been steadily decreasing from the initial value proposed by Hubble. If an alternative explanation for the red shift gains acceptance, static models could be back in contention [16].

\section{REFERENCES}

1. CANUTO, V.M. Variable G and the Strong Equivalence Principle, Int. J. Theor. Phys. 21 (1982), 633-663.

2. CANUTO, V. and HSIEH, S.-H. Comment on the paper 'A cosmological limit on the possible variation of $G^{\prime}$, Mon. Not. R. astr. Soc. 191 (1980), $605-606$.

3. CANUTO, V and HSIEH, S.-H. Cosmological Variation of $\mathrm{G}$ and the Solar Luminosity, Astrophys. J. 237 (1980), 613-615.

4. CANUTO, V., ADAMS, P.J., HSIEH, S.-H. and TSIANG, E. Scale-Covariant Theory of Gravitation and Astrophysical Applications, Phys.Rev.D. 16 (1977), 1643-1663.

5. WESSON, P.S. Gravity, Particles and Astrophysics, D. Reidel, Dordrecht (Holland), 1980.

6. WILL, C.M. The Confrontation between General Relativity and Experiment : An update, Phys. Rep. 113 (1984), 345-422.

7. CANUTO, V., HSIEH, S.-H. and ADAMS, P.J. Scale-Covariant Theory of Gravitation and Astrophysical Applications, Phys. Rev.Lett. 39 (1977), $429-432$.

8. ELLIS, G.F.R. Relativistic Cosmology, In General Relativity and Cosmology (Ed. R.K. Sachs), 104-182, Academic Press, New York, 1971.

9. ADAMS, P.J. Scale-Covariant Gravitation and Electromagnetism, Found. Phys. 9 (1979), 602-618.

10. CANUTO, V.M., HSIEH, S.-H. and OWEN, J.R. Scale Covariance and G-varying Cosmology. III, Astrophys. J. Suppl. 41 (1979), 263-300.

11. DIRAC, P.A.M. The Cosmological Constants, Nature 139 (1937), 323.

12. HELLINGS, R.W., ADAMS, P.J., ANDERSON, J.D., KEESEY, M.S., LAU, E.L. STANDISH, E.M., CANUTO, V.M. and GOLDMAN, I. Experimental Test of the Variability of G Using Viking Lander Ranging Data, Phys. Rev. Lett. 51 (1983), 1609-1612.

13. RAHMAN, H. and BANERJI, S. Can the Big-Bang Singularity be avoided in the Scale-Covariant Theory, Astrophys. Space Sci. 113 (1985), 405-412.

14. VAJK, J.P. Exact Robertson-Walker Cosmological Solutions Containing Relativistic Fluids, J. Math. Phys. 10 (1969), 1145-1151.

15. BEESHAM, A. Friedmann-Robertson-Walker Stiff Matter Solutions in the Scale Covariant Theory, Astrophys Space Sci. 120 (1986), 151-154.

16. BERMAN, M.S., SOMS, M.M. and GOMIDE, F.M. Brans-Dicke Static Universes, Gen. Rel. Grav. 21 (1989), 287-292. 


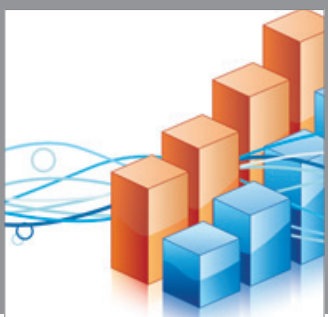

Advances in

Operations Research

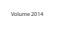

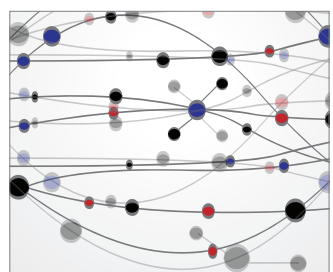

\section{The Scientific} World Journal
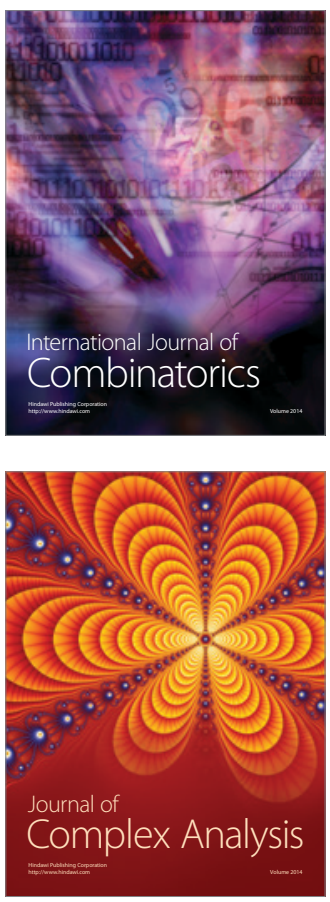

International Journal of

Mathematics and

Mathematical

Sciences
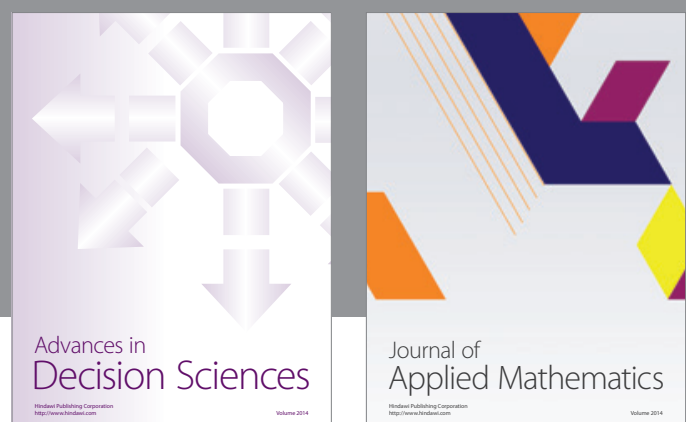

Journal of

Applied Mathematics
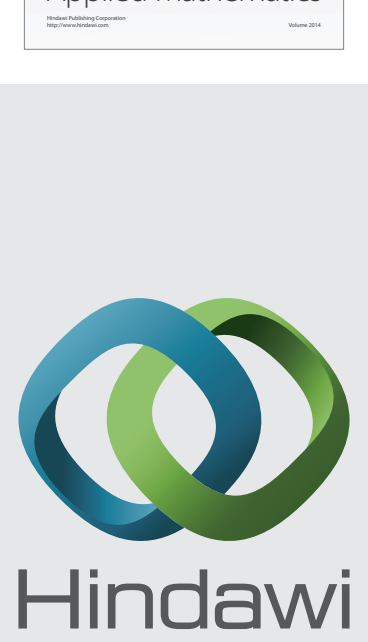

Submit your manuscripts at http://www.hindawi.com
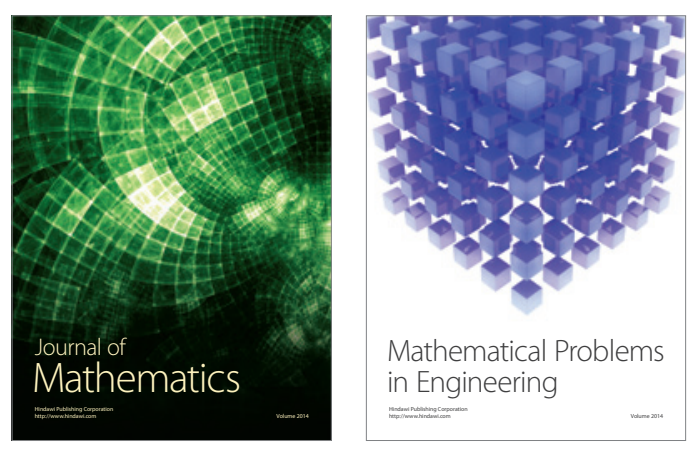

Mathematical Problems in Engineering
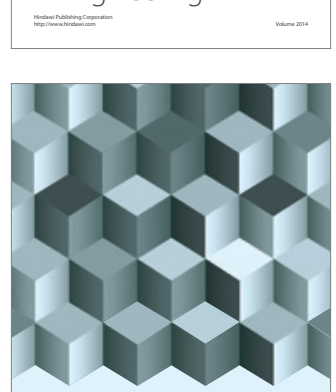

Journal of

Function Spaces
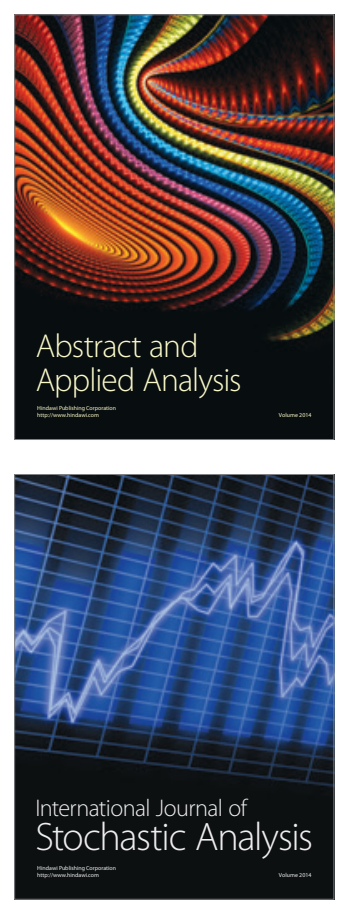

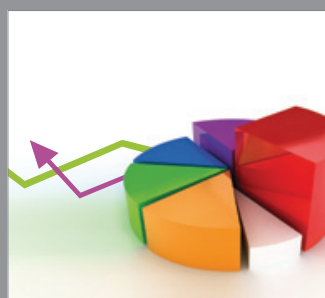

ournal of

Probability and Statistics

Promensencen
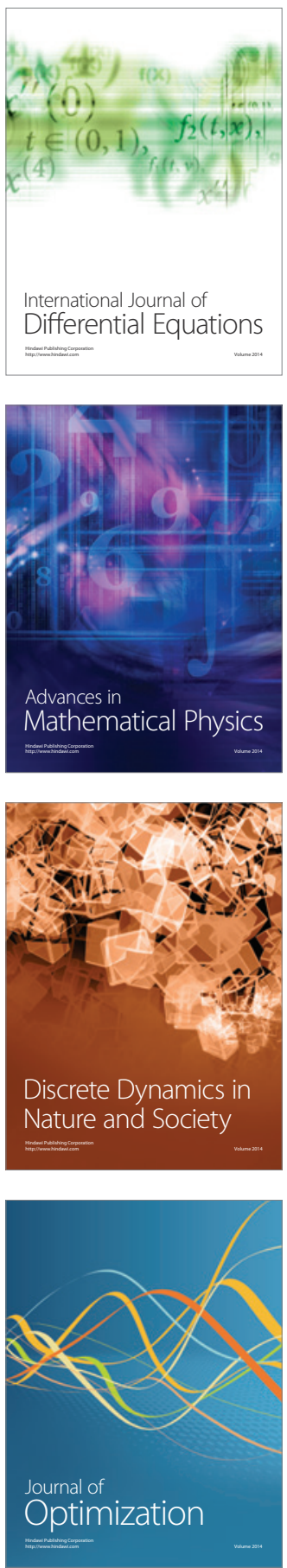